\title{
A Proof of the Gibbs-Thomson Formula in the Droplet Formation Regime ${ }^{1}$
}

\author{
Marek Biskup, ${ }^{2}$ Lincoln Chayes, ${ }^{2}$ and Roman Kotecký ${ }^{3}$
}

Received May 29, 2003; accepted September 3, 2003

\begin{abstract}
We study equilibrium droplets in two-phase systems at parameter values corresponding to phase coexistence. Specifically, we give a self-contained microscopic derivation of the Gibbs-Thomson formula for the deviation of the pressure and the density away from their equilibrium values which, according to the interpretation of the classical thermodynamics, appears due to the presence of a curved interface. The general - albeit heuristic - reasoning is corroborated by a rigorous proof in the case of the two-dimensional Ising lattice gas.
\end{abstract}

KEY WORDS: Gibbs-Thomson formula; droplet formation; Wulff construction; correlation inequality; cluster expansion.

\section{INTRODUCTION}

\subsection{The Problem}

The description of equilibrium droplets for systems with coexisting phases is one of the outstanding achievements of classical thermodynamics. Standard treatments of the subject highlight various formulæ relating the linear size of the droplet to a specific pressure difference. One of these, called the Gibbs-Thomson formula, concerns the difference between the actual pressure outside the droplet and the ambient pressure of the system without any droplets. (Or, in the terminology used in classical textbooks, "above a curved interface" and "above a planar interface," respectively.) The standard reasoning behind these formulæ is based primarily on macroscopic

\footnotetext{
${ }^{1}$ (C) 2003 by the authors. Reproduction, by any means, of the entire article for non-commercial purposes is permitted without charge.

${ }^{2}$ Department of Mathematics, UCLA, Los Angeles, California; e-mail: biskup@math.ucla.edu

${ }^{3}$ Center for Theoretical Study, Charles University, Prague, Czech Republic.
} 
concepts of pressure, surface tension, etc. But, notwithstanding their elegance and simplicity, these derivations do not offer much insight into the microscopic aspects of droplet equilibrium. The goal of the present paper is to give a self-contained derivation of the Gibbs-Thomson formula starting from the first principles of equilibrium statistical mechanics.

While straightforward on the level of macroscopic thermodynamics, an attempt for a microscopic theory of droplet equilibrium immediately reveals several technical problems. First of all, there is no obvious way-in equilibrium - to discuss finite-sized droplets that are immersed in an a priori infinite system. Indeed, the correct setting is the asymptotic behavior of finite systems that are scaling to infinity and that contain droplets whose size also scales to infinity (albeit, perhaps, at a different rate). Second, a statistical ensemble has to be produced whose typical configurations will feature an equilibrium droplet of a given linear size. A natural choice is the canonical ensemble with a tiny fraction of extra particles tuned so that a droplet of a given size is induced in the system. A difficulty here concerns the existence of a minimal droplet size as will be detailed below. Finally, for the specific problem at hand, the notions of pressure "above a curved interface" and "above a planar interface" have to be reformulated in terms of microscopic quantities which allow for a comparison of the difference between these pressures and the droplet size.

Some of these issues have previously been addressed by the present authors. Specifically, in refs. 4 and 5, we studied the droplet formation/ dissolution phenomena in the context of the canonical ensemble at parameters corresponding to phase coexistence and the particle density slightly exceeding the ambient limiting rarefied density. It was found that, if $V$ is the volume of the system and $\delta N$ is the particle excess, droplets form when the ratio $(\delta N)^{(d+1) / d} / V$ is of the order of unity. In particular, there exists a dimensionless parameter $\Delta$, proportional to the thermodynamic limit of this ratio, and a non-trivial critical value $\Delta_{\mathrm{c}}$, such that, for $\Delta<\Delta_{\mathrm{c}}$, all of the excess will be absorbed into the (Gaussian) fluctuations of the ambient gas, while if $\Delta>\Delta_{\mathrm{c}}$, a mesoscopic droplet will form. Moreover, the droplet will only subsume a fraction $\lambda_{\Delta}<1$ of the excess particles. This fraction gets smaller as $\Delta$ decreases to $\Delta_{\mathrm{c}}$, yet the minimum fraction $\lambda_{\Delta_{\mathrm{c}}}$ does not vanish. It is emphasized that these minimum sized droplets are a mesoscopic phenomenon: The linear size of the droplet will be proportional to $V^{1 /(d+1)} \ll V^{1 / d}$ and the droplet thus occupies a vanishing fraction of the system. Note that the total volume cannot be taken arbitrary large if there is to be a fixed-size droplet at all.

The droplet formation/dissolution phenomena have been the subject of intensive study in last few years. The fact that $d /(d+1)$ is the correct exponent for the scale on which droplets first appear was shown rigorously 
in ref. 15 (see also ref. 21); a heuristic derivation may go back at least to ref. 3. The existence of a sharp minimal droplet size on the scale $V^{1 /(d+1)}$ was described in ref. 22, more recently in refs. 4 and 25 and yet again in ref. 2. In the context of the 2D Ising system, a rigorous justification of the theory outlined in ref. 4 was provided in ref. 5 . We note that the existence of a minimal droplet size seems to be ultimately related to the pressure difference "due" to the presence of a droplet as expressed by the Gibbs-Thomson formula. Indeed, from another perspective (which is more or less that of refs. 22 and 25), the formation/dissolution phenomena can be understood on the basis of arguments in which the Gibbs-Thomson formula serves as a foundation. Finally, we remark that although the generation of droplets is an inherently dynamical phenomenon (beyond the reach of current methods) it is possible that, on limited temporal and spatial scales, the equilibrium asymptotics is of direct relevance.

The remainder of this paper is organized as follows. In the next subsection (Section 1.2) we will present an autonomous derivation of the Gibbs-Thomson formula based on first principles of statistical mechanics. Aside from our own (modest) appreciation of this approach, Section 1.2 is worthwhile in the present context because the rigorous analysis develops precisely along these lines. In Section 2, we will restrict our attention to the 2D Ising lattice gas, define explicitly the relevant quantities and present our rigorous claims in the form of mathematical theorems. The proofs will come in Section 3.

\subsection{Heuristic Derivation}

Let us consider a two-phase system at parameter values corresponding to phase coexistence. We will assume that the two phases are distinguished by their densities and, although the forthcoming derivation is completely general, we will refer to the dense phase as liquid and to the rarefied phase as gas. Confining the system to a $(d \geqslant 2)$-dimensional volume $V$, we will consider a canonical ensemble at inverse temperature $\beta$ and the number of particles fixed to the value

$$
N=\rho_{\mathrm{g}} V+\left(\rho_{\ell}-\rho_{\mathrm{g}}\right) \delta V .
$$

Here, $\rho_{\ell}$ and $\rho_{\mathrm{g}}$ are the bulk densities of the liquid and gas, respectively, and the particle excess is $\delta N=\left(\rho_{\ell}-\rho_{\mathrm{g}}\right) \delta V$ with $\delta V \ll V$. Let $w_{1}$ denote the dimensionless interfacial free energy (expressed in multiples of $\beta^{-1}$ ), which represents the cost of an optimally-shaped droplet of unit volume, and let $x$ denote the response function, $x=\frac{1}{V}\left\langle(N-\langle N\rangle)^{2}\right\rangle$, which is 
essentially the isothermal compressibility. Then, as has been argued in ref. 4 , if the parameter

$$
\Delta=\frac{\left(\rho_{\ell}-\rho_{\mathrm{g}}\right)^{2}}{2 \varkappa w_{1}} \frac{(\delta V)^{\frac{d+1}{d}}}{V},
$$

is less than a critical value $\Delta_{\mathrm{c}}=\frac{1}{d}\left(\frac{d+1}{2}\right)^{\frac{d+1}{d}}$, all of the particle excess will be absorbed by the background fluctuations, while, for $\Delta>\Delta_{\mathrm{c}}$, a fraction of the excess particles will condense into a droplet. Moreover, the volume of this droplet will be (in the leading order) $\lambda_{\Delta} \delta V$, where $\lambda_{\Delta} \in[0,1]$ is the maximal solution to the equation

$$
\frac{d-1}{d} \lambda^{-1 / d}=2 \Delta(1-\lambda)
$$

Note that $\lambda_{\Delta_{\mathrm{c}}}=2 /(d+1)$ as advertised; that is to say, the droplet does not appear gradually. Furthermore, as is of interest in certain anisotropic situations where the droplet plays a role of an equilibrium crystal, the droplet has a particular shape, known as the Wulff shape, which optimizes the overall interfacial free energy for a given volume.

Gibbs-Thomson I: The Density. On the basis of the aforementioned claims, we can already state a version of the Gibbs-Thomson formula for the difference of densities "due to the presence of a curved interface." Indeed, since the droplet only accounts for a fraction, $\lambda_{\Delta}$, of the excess particles, the remainder $\left(1-\lambda_{\Delta}\right)\left(\rho_{\ell}-\rho_{\mathrm{g}}\right) \delta V$, of these particles reside in the bulk. Supposing that the droplet subsumes only a negligible fraction of the entire volume, i.e., $\delta V \ll V$, the gas surrounding the droplet will thus have the density

$$
\bar{\rho}_{\mathrm{g}}=\rho_{\mathrm{g}}+\left(1-\lambda_{\Delta}\right)\left(\rho_{\ell}-\rho_{\mathrm{g}}\right) \frac{\delta V}{V}(1+o(1))
$$

Here $o(1)$ is a quantity tending to zero as $V$ tends to infinity while keeping $\Delta$ finite (and $\Delta>\Delta_{\mathrm{c}}$ ). Invoking (1.2) and (1.3), this is easily converted into

$$
\bar{\rho}_{\mathrm{g}}=\rho_{\mathrm{g}}+\frac{d-1}{d} \frac{\varkappa w_{1}}{\rho_{\ell}-\rho_{\mathrm{g}}} \frac{1}{\left(\lambda_{\Delta} \delta V\right)^{1 / d}}(1+o(1)) .
$$

Thus, the density of the gas surrounding the droplet will exceed the density of the ambient gas by a factor inversely-proportional to the linear size of the droplet. This is (qualitatively) what is stated by the Gibbs-Thomson formula. 
In order to make correspondence with physics literature, let us assume that the droplet is spherical - which is the case for an isotropic surface tension. Then we have

$$
w_{1}=\beta \sigma S_{d}\left(\frac{S_{d}}{d}\right)^{-\frac{d-1}{d}} \quad \text { and } \quad \lambda_{\Delta} \delta V=\frac{S_{d}}{d} r^{d}
$$

where $\sigma$ is the surface tension, $S_{d}$ is the surface area of a unit sphere in $\mathbb{R}^{d}$ and $r$ is the radius of the droplet. Substituting these relations into (1.5), we will get

$$
\bar{\rho}_{\mathrm{g}}=\rho_{\mathrm{g}}+(d-1) \frac{\beta \sigma \varkappa}{\rho_{\ell}-\rho_{\mathrm{g}}} \frac{1}{r}(1+o(1)) .
$$

Of course, all three formulas (1.4), (1.5), and (1.7) represent the leading order asymptotic in $1 / r$. Higher-order corrections go beyond the validity of the presented argument.

Remark 1.1. We note that equation (1.7) differs from the usual corresponding version of the Gibbs-Thomson formula in which the $x$ appearing above is replaced by $\rho_{\mathrm{g}}$. This is due to the approximation $\varkappa \approx \rho_{\mathrm{g}}$ which is justified only in the ideal-gas limit of the rarefied phase.

Pressures above Curved/Planar Interfaces. Next we turn our attention to the Gibbs-Thomson formula for the pressure. Here we immediately run into a complication; while the density is a well-defined object in finite volume, the pressure, by its nature, is a macroscopic commodity. Thus, strictly speaking, the pressure should be discussed in the context of thermodynamic limits.

In the present context we need to define the "pressure of the gas surrounding a droplet." In order to do so, we will consider two canonical ensembles with the same number of particles given by (1.1), in volumes $V$ and $V+\Delta V$, where $\Delta V \ll V$. From the perspective of equilibrium thermodynamics, these two situations describe the initial and terminal states of the gas undergoing isothermal expansion. Standard statistical-mechanical formulas tell us that the change of the relevant thermodynamic potential (the Helmholtz free energy) during this expansion is given as the pressure times the difference of the volumes $\Delta V$. Using $Z_{\mathrm{C}}(N, V)$ to denote the canonical partition function of $N$ particles in volume $V$, we thus define the relevant pressure $p_{V}$ by

$$
p_{V}=\frac{1}{\beta} \frac{1}{\Delta V} \log \frac{Z_{\mathrm{C}}\left(\rho_{\mathrm{g}} V+\left(\rho_{\ell}-\rho_{\mathrm{g}}\right) \delta V, V+\Delta V\right)}{Z_{\mathrm{C}}\left(\rho_{\mathrm{g}} V+\left(\rho_{\ell}-\rho_{\mathrm{g}}\right) \delta V, V\right)} .
$$


For finite $V, \Delta V$, etc., the quantity $p_{V}$ still depends on $\Delta V$. As it turns out, this dependence (which we will refrain from making notationally explicit) will annul in any limit $V, \Delta V \rightarrow \infty$ with $\Delta V / \partial V \rightarrow 0$, where $\partial V$ denotes the boundary of $V$. However, we must consider a limiting procedure for which $\triangle V$ also does not "disturb" the droplet. This is a slightly delicate subject matter to which we will return shortly.

Our next goal is to give a mathematical interpretation of the pressure "above a planar interface." As it turns out (and as is the standard in all derivations), here the correct choice is to take simply the pressure of the ambient gas phase. (See Remark 1.3 for further discussions.) Using $Z_{\mathrm{G}}(\mu, V)$ to denote the grand canonical partition function, with $\mu$ denoting the chemical potential, this quantity is defined by the (thermodynamic) limit

$$
p_{\infty}=\frac{1}{\beta} \lim _{V \rightarrow \infty} \frac{1}{V} \log Z_{\mathrm{G}}\left(\mu_{\mathrm{t}}, V\right),
$$

Here we have prepositioned the chemical potential to the transitional value, i.e., $\mu=\mu_{\mathrm{t}}$. By well-known arguments, this limit is independent of how $V$ tends to infinity provided $\partial V / V$ tends to zero as $V \rightarrow \infty$.

Since we are ultimately looking for an expression for the difference $p_{V}-p_{\infty}$, instead of (1.9) we would rather have an expression that takes a form similar to (1.8). We might try to use the fact that $\log Z_{\mathrm{G}}\left(\mu_{\mathrm{t}}, V\right)=$ $\beta p_{\infty} V+O(\partial V)$, but then the boundary term will be much larger than the actual Gibbs-Thomson correction. We thus have to develop a more precise representation of the grand canonical partition function. For simplicity, we will restrict ourselves to the cases when $V$ is a rectangular box, in which case we expect to have

$$
\log Z_{\mathrm{G}}\left(\mu_{\mathrm{t}}, V\right)=\beta p_{\infty} V+\tau_{\text {wall }} \partial V+O\left(V^{\frac{d-2}{d}}\right) .
$$

Here $\tau_{\text {wall }}$ denotes a wall surface tension which depends on the boundary conditions. The error term represents the contribution from lower-dimensional facets of $V$, e.g., edges and corners of $V$ in $d=3$. Such a representation can be justified using low-temperature expansions, see ref. 8 , and/or by invoking rapid decay of correlations. Of course, this will be discussed in excruciating detail in Section 3 of the present paper.

Using the representation (1.10), we can now write

$$
\beta p_{\infty}=\frac{1}{\Delta V} \log \frac{Z_{\mathrm{G}}\left(\mu_{\mathrm{t}}, V+\Delta V\right)}{Z_{\mathrm{G}}\left(\mu_{\mathrm{t}}, V\right)}+O\left(\frac{\partial(V+\Delta V)-\partial V+V^{\frac{d-2}{d}}}{\Delta V}\right),
$$

which supposes that both $V$ and $V+\Delta V$ are rectangular volumes. 
Our goal is to limit $\Delta V$ to the values for which the error term is negligible compared with the anticipated Gibbs-Thomson correction. First, supposing that $\Delta V \ll V$, we find that the difference $\partial(V+\Delta V)-\partial V$ is of the order $\Delta V / V^{1 / d}$. Second, assuming that $\Delta$ from (1.2) is finite and exceeding $\Delta_{\mathrm{c}}$ (which is necessary to have any droplet at all), we have $\delta V \sim V^{d /(d+1)}$. These two observations show that the contribution of $\partial(V+\Delta V)-\partial V$ to the error term in (1.11) is indeed negligible compared with $(\delta V)^{-1 / d}$. A similar calculation shows that the the second part of the error term, $V^{(d-2) / d} / \Delta V$, on the right-hand side of (1.11) is negligible compared with $(\delta V)^{-1 / d}$ provided that

$$
\triangle V \gg V^{\frac{d-2}{d}+\frac{1}{d+1}} \text {. }
$$

It is easy to check - see formula (1.23) - that (1.12) can be satisfied while maintaining $\Delta V \ll \delta V$. This observation will be essential in the forthcoming developments.

The formulas (1.8-1.11) can be conveniently subtracted in terms of the probability $\mathbb{P}_{V}(N)$ that, in the grand canonical ensemble, there are exactly $N$ particles in volume $V$. Explicitly, denoting

$$
\mathbb{P}_{V}(N)=\frac{e^{\beta \mu_{\mathrm{t}} N} Z_{\mathrm{C}}(N, V)}{Z_{\mathrm{G}}\left(\mu_{\mathrm{t}}, V\right)},
$$

we get

$$
\beta\left(p_{V}-p_{\infty}\right)=\frac{1}{\Delta V} \log \frac{\mathbb{P}_{V+\Delta V}\left(\rho_{\mathrm{g}} V+\left(\rho_{\ell}-\rho_{\mathrm{g}}\right) \delta V\right)}{\mathbb{P}_{V}\left(\rho_{\mathrm{g}} V+\left(\rho_{\ell}-\rho_{\mathrm{g}}\right) \delta V\right)}+o\left((\delta V)^{-1 / d}\right) .
$$

Here we have applied (1.12) to simplify the error term.

Gibbs-Thomson II: The Pressure. Now we are in a position to derive the desired Gibbs-Thomson formula for the pressure. A principal tool for estimating the ratio of the probabilities in (1.14) will be another result of ref. 4 which tells us that, in the limit $V \rightarrow \infty$,

$$
-\log \mathbb{P}_{V}\left(\rho_{\mathrm{g}} V+\left(\rho_{\ell}-\rho_{\mathrm{g}}\right) \delta V\right)=w_{1}(\delta V)^{\frac{d-1}{d}}\left(\Phi_{\Delta}^{\star}+o(1)\right),
$$

where $\Phi_{\Delta}^{\star}$ is the absolute minimum of the function

$$
\Phi_{\Delta}(\lambda)=\lambda^{\frac{d-1}{d}}+\Delta(1-\lambda)^{2}
$$

on $[0,1]$. Since $\rho_{\mathrm{g}} V+\left(\rho_{\ell}-\rho_{\mathrm{g}}\right) \delta V=\rho_{\mathrm{g}}(V+\Delta V)+\left(\rho_{\ell}-\rho_{\mathrm{g}}\right)(\alpha \delta V)$, where

$$
\alpha=1-\frac{\rho_{\mathrm{g}}}{\rho_{\ell}-\rho_{\mathrm{g}}} \frac{\Delta V}{\delta V}
$$


we also have, again in the limit $V \rightarrow \infty$,

$$
-\log \mathbb{P}_{V+\Delta V}\left(\rho_{\mathrm{g}} V+\left(\rho_{\ell}-\rho_{\mathrm{g}}\right) \delta V\right)=w_{1}(\alpha \delta V)^{\frac{d-1}{d}}\left(\Phi_{\Delta(\alpha)}^{\star}+o(1)\right),
$$

where we have introduced the shorthand $\Delta(\alpha)=\alpha^{\frac{d+1}{d}} \Delta$.

Supposing that $\Delta V \ll \delta V$, we can write

$$
\Phi_{\Delta(\alpha)}^{\star}=\Phi_{\Delta}^{\star}-\frac{\rho_{\mathrm{g}}}{\rho_{\ell}-\rho_{\mathrm{g}}} \frac{\Delta V}{\delta V}\left(1-\lambda_{\Delta}\right)^{2}+o(\Delta V / \delta V)
$$

and thus, to the leading order in $\Delta V / \delta V$,

$$
\begin{aligned}
& \beta\left(p_{V}-p_{\infty}\right) \\
& \quad=w_{1} \frac{\rho_{\mathrm{g}}}{\rho_{\ell}-\rho_{\mathrm{g}}} \frac{1}{(\delta V)^{1 / d}}\left[\frac{d-1}{d} \Phi_{\Delta}^{\star}+\frac{d+1}{d} \Delta\left(1-\lambda_{\Delta}\right)^{2}+o(1)\right] .
\end{aligned}
$$

After some manipulations involving (1.16) and (1.3), the square bracket on the right-hand side turns out to equal $\frac{d-1}{d} \lambda_{\Delta}^{-1 / d}+o(1)$. Thus we finally derive

$$
\beta\left(p_{V}-p_{\infty}\right)=\frac{d-1}{d} \frac{w_{1} \rho_{\mathrm{g}}}{\rho_{\ell}-\rho_{\mathrm{g}}} \frac{1}{\left(\lambda_{\Delta} \delta V\right)^{1 / d}}(1+o(1)) .
$$

In the case of an isotropic surface tension, formula (1.21) again reduces to

$$
p_{V}-p_{\infty}=(d-1) \frac{\sigma \rho_{\mathrm{g}}}{\rho_{\ell}-\rho_{\mathrm{g}}} \frac{1}{r}(1+o(1)) .
$$

This is the (leading order) Gibbs-Thomson correction; the one which is usually derived ${ }^{(24,29)}$ by invoking thermodynamic considerations. We note that here the gas-density $\rho_{\mathrm{g}}$ in the numerator is fully justified, cf. Remark 1.1.

Remark 1.2. We note that higher orders in $1 / r$ - as predicted by the "exponential" Gibbs-Thomson formula in classical thermodynamics-go beyond the validity of the formulas (1.15) and (1.18). In fact, as a closer look at the $V$-dependence of $\delta V$ and $\partial V$ suggests, these corrections may depend on the choice of the volumes $V$ and $V+\Delta V$ and on the boundary condition. We further remark that both formulas (1.5) and (1.21) have been derived for the situation when a droplet of the dense phase forms inside the low-density phase. However, a completely analogous derivation works for a droplet of a low-density phase immersed in a high-density environment (e.g., vapor bubbles in water). 
Remark 1.3. Once we have derived the Gibbs-Thomson formula (1.21), we can also justify our choice of $p_{\infty}$ for the pressure "above a planar interface." First let us note that, in (1.21), $p_{\infty}$ can be viewed as a convenient normalization constant - subtracting (1.21) for two different volumes, say $V_{1}$ and $V_{2}$, the quantity $p_{\infty}$ completely factors out. Moreover, if $V_{1} \ll V_{2}$, the contribution of the droplet in $V_{2}$ to such a difference will be negligible. Thus, in the limit when $V_{2} \rightarrow \infty$ and $V_{1}$ stays fixed, $p_{V_{1}}-p_{V_{2}}$ tends to $p_{V_{1}}-p_{\infty}$ as expressed in (1.21). Since also the droplet in $V_{2}$ becomes more and more flat in this limit, $p_{\infty}$ indeed represents the pressure "above a planar interface."

This concludes our heuristic derivation of the Gibbs-Thomson formula. We reiterate that all of the above only makes good sense when $\Delta V$ has been chosen such that

$$
V^{1-\frac{2}{d}+\frac{1}{d+1}} \ll \Delta V \ll \delta V \sim V^{1-\frac{1}{d+1}} .
$$

As is easily checked, these inequalities represent a non-trivial interval of values of $\Delta V$. In the next sections, where we will rigorously treat the case of the two-dimensional Ising lattice gas, the inequality on the right-hand side will be guaranteed by taking $\Delta V=\eta \delta V$ and then performing the limits $V \rightarrow \infty$ followed by $\eta \rightarrow 0$.

\section{RIGOROUS RESULTS}

\subsection{The Model}

Throughout the remainder of this paper, we will focus our attention on the two-dimensional Ising lattice gas. The latter refers to a system where each site of the square lattice $\mathbb{Z}^{2}$ can be either vacant or occupied by one particle. The state of each site is characterized by means of an occupation number $n_{x}$ which is zero for a vacant site and one for an occupied site. The formal Hamiltonian of the system can be written as

$$
\mathscr{H}=-\sum_{\langle x, y\rangle} n_{x} n_{y}-\mu \sum_{x} n_{x}
$$

Here $\langle x, y\rangle$ denotes a nearest-neighbor pair on $\mathbb{Z}^{2}$ and $\mu$ plays the role of a chemical potential. Note that the Hamiltonian describes particles with a hard-core repulsion and short-range attraction (with coupling constant set to unity).

The Gibbs measure (or Gibbs state) on particle configurations in a finite volume $\Lambda \subset \mathbb{Z}^{2}$ is defined using the finite-volume version of (2.1) and 
a boundary condition on the boundary of $\Lambda$. Explicitly, let $\partial \Lambda$ be the set of sites in $\mathbb{Z}^{2} \backslash \Lambda$ that have a bond into $\Lambda$ and let $\mathscr{H}_{\Lambda}$ be the restriction of $\mathscr{H}$ obtained by considering only pairs $\{x, y\} \cap A \neq \varnothing$ in the first sum in (2.1) and sites $x \in \Lambda$ in the second sum. If $n_{\Lambda} \in\{0,1\}^{\Lambda}$ is a configuration in $\Lambda$ and $n_{\partial \Lambda}$ is a boundary condition (i.e., a configuration on the boundary $\partial \Lambda$ of $\Lambda$ ), and if $\mathscr{H}_{\Lambda}\left(n_{\Lambda} \mid n_{\partial \Lambda}\right)$ is the Hamiltonian for these two configurations, then the probability of $n_{A}$ in the corresponding Gibbs measure is given by

$$
P_{\Lambda}^{n_{\partial \Lambda}, \beta, \mu}\left(n_{\Lambda}\right)=\frac{e^{-\beta \mathscr{H}_{\Lambda}\left(n_{\Lambda} \mid n_{\partial \Lambda}\right)}}{Z_{\mathrm{G}}^{n_{\partial \Lambda}, \beta}(\mu, \Lambda)} .
$$

Here, as usual, $\beta \geqslant 0$ is the inverse temperature and the normalization constant, $Z_{\mathrm{G}}^{n_{\partial}}, \beta(\mu, \Lambda)$, is the grand canonical partition function in $\Lambda$ corresponding to the boundary condition $n_{\partial \Lambda}$. We recall that, according to the standard DLR-scheme, ${ }^{(16)}$ the system is at phase coexistence if (depending on the boundary conditions and/or the sequence of volumes) there is more than one infinite-volume limit of the measures in (2.2). Of particular interest in this work will be the measure in $L \times L$ rectangular volumes $\Lambda_{L} \subset \mathbb{Z}^{2}$ with vacant (i.e., $n_{\partial \Lambda_{L}} \equiv 0$ ) boundary condition. In this case we will denote the object from (2.2) by $P_{L}^{\circ, \beta, \mu}$.

As is well known, the lattice gas model (2.1) is equivalent to the Ising magnet with the (formal) Hamiltonian

$$
\mathscr{H}=-J \sum_{\langle x, y\rangle} \sigma_{x} \sigma_{y}-h \sum_{x} \sigma_{x},
$$

coupling constant $J=1 / 4$, external field $h=\mu-2$ and the Ising spins $\left(\sigma_{x}\right)$ related to the occupation variables $\left(n_{x}\right)$ via $\sigma_{x}=2 n_{x}-1$. The \pm -symmetry of the Ising model also allows us to identify the regions of phase coexistence of the lattice gas model defined by (2.1): There is a value $\beta_{\mathrm{c}}=2 \log (1+\sqrt{2})$ of the inverse temperature such that for $\beta>\beta_{\mathrm{c}}$ and $\mu=\mu_{\mathrm{t}}=2$, there exist two distinct translation-invariant, extremal, ergodic, infinite-volume Gibbs states for the Hamiltonian (2.1) - a "liquid" state characterized by an abundance of particles over vacancies and a "gaseous" state, characterized by an abundance of vacancies over occupied sites. In the Ising-spin language, these states correspond to the plus and minus states which in the lattice gas language translate to the states generated by the fully occupied or vacant boundary conditions. We will use $\langle-\rangle_{\beta}^{\circ}$ and $\langle-\rangle_{\beta}^{\bullet}$ to denote the expectation with respect to the (infinite-volume) "gaseous" and "liquid" state, respectively. 
In order to discuss the Gibbs-Thomson formula in this model, we need to introduce the relevant quantities. Assuming $\mu=\mu_{\mathrm{t}}$ and $\beta>\beta_{\mathrm{c}}$, we will begin by defining the gas and liquid densities:

$$
\rho_{\mathrm{g}}=\rho_{\mathrm{g}}(\beta)=\left\langle n_{0}\right\rangle_{\beta}^{\circ} \quad \text { and } \quad \rho_{\ell}=\rho_{\ell}(\beta)=\left\langle n_{0}\right\rangle_{\beta}^{\bullet},
$$

where $n_{0}$ refers to the occupation variable at the origin. Note that, by the plus-minus Ising symmetry, $\left\langle n_{0}\right\rangle_{\beta}^{\circ}=\left\langle 1-n_{0}\right\rangle_{\beta}^{\bullet}$ and thus $\rho_{\ell}+\rho_{\mathrm{g}}=1$. Next we will introduce the quantity $\varkappa$ which is related to isothermal compressibility:

$$
\varkappa=\sum_{x \in \mathbb{Z}^{2}}\left(\left\langle n_{0} n_{x}\right\rangle_{\beta}^{\circ}-\rho_{\mathrm{g}}^{2}\right) .
$$

The sum converges for all $\beta>\beta_{\mathrm{c}}$ by the exponential decay of truncated particle-particle correlations, $\left|\left\langle n_{x} n_{y}\right\rangle_{\beta}^{\circ}-\rho_{\mathrm{g}}^{2}\right| \leqslant e^{-|x-y| / \xi}$, where $\xi=\xi(\beta)<\infty$ denotes the correlation length. The latter was proved in refs. 12 and 28 in the context of the 2D Ising model.

The last object we need to bring into play is the surface tension or the interfacial free energy. In the 2D Ising model, one can use several equivalent definitions. Since we will not need any of them explicitly, it suffices if we just summarize the major concepts as formulated, more or less, in refs. 14 and 26: First, for each $\beta>\beta_{\mathrm{c}}$, there is a continuous function $\tau_{\beta}:\left\{\mathbf{n} \in \mathbb{R}^{2}:|\mathbf{n}|=1\right\} \rightarrow(0, \infty)$, called the microscopic surface tension. Roughly speaking, $\tau_{\beta}(\mathbf{n})$ is the cost per length of an interface with normal vector $\mathbf{n}$ that separates a "gaseous" and "liquid" region.

This allows to introduce the so called Wulff functional $\mathscr{W}_{\beta}$ that assigns to each rectifiable curve $\varphi=\left(\varphi_{t}\right)$ in $\mathbb{R}^{2}$ the value

$$
\mathscr{W}_{\beta}(\varphi)=\int_{\varphi} \tau_{\beta}\left(\mathbf{n}_{t}\right) \mathrm{d} \mathbf{n}_{t}
$$

Here $\mathbf{n}_{t}$ is the normal vector to $\varphi$ at the point $\varphi_{t}$.

The quantity $\mathscr{W}_{\beta}(\partial D)$ expresses the macroscopic cost of a droplet $D$ with boundary $\partial D$. Indeed, as has been established in the course of last few years, ${ }^{(14,19-21,26,27)}$ the probability in the measure $P_{L}^{\circ}, \beta, \mu_{\mathrm{t}}$ that a droplet of "liquid" phase occurs whose shape is "near" that of the set $D$ is given, to leading order, by $\exp \left\{-\mathscr{W}_{\beta}(\partial D)\right\}$. Thus the "most favorable" droplet shape is obtained by minimizing $\mathscr{W}_{\beta}(\partial D)$ over all $D$ with a given volume. Using $W$ to denote the minimizing set with a unit volume (which can be explicitly constructed $\left.{ }^{(13,17,30)}\right)$, we define

$$
w_{1}(\beta)=\mathscr{W}_{\beta}(\partial W) .
$$


By well-known properties of the surface tension, we have $w_{1}(\beta)>0$ once $\beta>\beta_{\mathrm{c}}$. We note that, as in the heuristic section, the customary factor $1 / \beta$ is incorporated into $\tau_{\beta}$ in our definition of the surface tension.

Remark 2.1. For those more familiar with the magnetic terminology, let us pause to identify the various quantities in Ising language: First, if $m^{\star}(\beta)$ is the spontaneous magnetization, then we have $\rho_{\mathrm{g}}(\beta)=$ $\frac{1}{2}\left(1-m^{\star}(\beta / 4)\right)$ and $\rho_{\ell}(\beta)=\frac{1}{2}\left(1+m^{\star}(\beta / 4)\right)$. Similarly, if $\chi(\beta)$ denotes the magnetic susceptibility in the Ising spin system, then $\chi(\beta)=x(\beta / 4) / 4$. Finally, the quantity $w_{1}(\beta)$ corresponds exactly to the similar quantity for the spin system at a quarter of the inverse temperature.

\subsection{Known Facts}

Here we will review some of the rigorous results concerning the 2D Ising lattice gas in a finite volume and a fixed number of particles. In the language of statistical mechanics, this corresponds to the canonical ensemble. The stated theorems are transcribes of the corresponding results from ref. 5 .

Recall our notation $P_{L}^{\circ, \beta, \mu}$ for the Gibbs measure in $L \times L$ rectangular box $\Lambda_{L}$ and vacant boundary conditions on $\partial \Lambda_{L}$. Let $\left(v_{L}\right)$ be a sequence of positive numbers tending to infinity in such a way that $v_{L}^{3 / 2} /\left|\Lambda_{L}\right|$ tends to a finite non-zero limit. In addition, suppose that $\left(v_{L}\right)$ is such that $\rho_{\mathrm{g}}\left|\Lambda_{L}\right|+\left(\rho_{\ell}-\rho_{\mathrm{g}}\right) v_{L}$ is a number from $\left\{0,1, \ldots,\left|\Lambda_{L}\right|\right\}$ for all $L$. For any configuration $\left(n_{x}\right)$ in $\Lambda_{L}$, let $N_{L}$ denote the total number of particles in $\Lambda_{L}$, i.e.,

$$
N_{L}=\sum_{x \in \Lambda_{L}} n_{x}
$$

Our first theorem concerns the large-deviation asymptotic for the random variable $N_{L}$. The following is a rigorous version of the claim (1.15), which, more or less, is Theorem 1.1 from ref. 5.

Theorem A. Let $\beta>\beta_{\mathrm{c}}$ and let the sequence $\left(v_{L}\right)$ and the quantities $\rho_{\mathrm{g}}=\rho_{\mathrm{g}}(\beta), \rho_{\ell}=\rho_{\ell}(\beta), \varkappa=\varkappa(\beta)$, and $w_{1}=w_{1}(\beta)$ be as defined previously. Suppose that the limit

$$
\Delta=\frac{\left(\rho_{\ell}-\rho_{\mathrm{g}}\right)^{2}}{2 \varkappa w_{1}} \lim _{L \rightarrow \infty} \frac{v_{L}^{3 / 2}}{\left|\Lambda_{L}\right|}
$$


exists with $\Delta \in(0, \infty)$. Then

$$
\begin{aligned}
\lim _{L \rightarrow \infty} & \frac{1}{\sqrt{v_{L}}} \log P_{L}^{\circ}, \beta, \mu_{\mathrm{t}}\left(N_{L}=\rho_{\mathrm{g}}\left|\Lambda_{L}\right|+\left(\rho_{\ell}-\rho_{\mathrm{g}}\right) v_{L}\right) \\
& =-w_{1} \inf _{0 \leqslant \lambda \leqslant 1} \Phi_{\Delta}(\lambda),
\end{aligned}
$$

where $\Phi_{\Delta}(\lambda)=\sqrt{\lambda}+\Delta(1-\lambda)^{2}$.

We proceed by a description of the typical configurations in the conditional measure

$$
P_{L}^{\circ, \beta, \mu_{\mathrm{t}}}\left(\cdot\left|N_{L}=\rho_{\mathrm{g}}\right| \Lambda_{L} \mid+\left(\rho_{\ell}-\rho_{\mathrm{g}}\right) v_{L}\right),
$$

which, we note, actually does not depend on the choice of the chemical potential. Our characterization will be based on the notion of Peierls' contours: Given a particle configuration, let us place a dual bond in the middle of each direct bond connecting an occupied and a vacant site. These dual bonds can be connected into self-avoiding polygons by applying an appropriate "rounding rule," as discussed in ref. 14 and illustrated in, e.g., Fig. 1 of ref. 5. Given a contour $\gamma$, let $V(\gamma)$ denote the set of sites enclosed by $\gamma$. In accord with ref. 5, we also let diam $\gamma$ denote the diameter of the set $V(\gamma)$ in the $\ell_{2}$ metric on $\mathbb{Z}^{2}$. If $\Gamma$ is a collection of contours, we say that $\gamma \in \Gamma$ is an external contour if it is not surrounded by any other contour from $\Gamma$.

While "small" contours are just natural fluctuations within a given phase, "large" contours should somehow be interpreted as droplets. It turns out that the corresponding scales are clearly separated with no intermediate contours present in typical configurations. The following is essentially the content of Theorem 1.2 and Corollary 1.3 from ref. 5 .

Theorem B. Let $\beta>\beta_{\mathrm{c}}$ and let the sequence $\left(v_{L}\right)$ and the quantities $\rho_{\mathrm{g}}=\rho_{\mathrm{g}}(\beta), \rho_{\ell}=\rho_{\ell}(\beta), \chi=\chi(\beta)$, and $w_{1}=w_{1}(\beta)$ be as defined previously. Suppose that the limit in (2.9) exists with $\Delta \in(0, \infty)$ and let $\Delta_{\mathrm{c}}=\frac{1}{2}(3 / 2)^{3 / 2}$. There exists a number $K=K(\beta, \Delta)<\infty$ such that, for each $\epsilon>0$ and $L \rightarrow \infty$, the following holds with probability tending to one in the distribution (2.11):

(1) If $\Delta<\Delta_{\mathrm{c}}$, then all contours $\gamma$ satisfy diam $\gamma \leqslant K \log L$.

(2) If $\Delta>\Delta_{\mathrm{c}}$, then there exists a unique contour $\gamma_{0}$ with

$$
\lambda_{\Delta} v_{L}(1-\epsilon) \leqslant\left|V\left(\gamma_{0}\right)\right| \leqslant \lambda_{\Delta} v_{L}(1+\epsilon)
$$


and

$$
\rho_{\ell} \lambda_{\Delta} v_{L}(1-\epsilon) \leqslant \sum_{x \in V\left(\gamma_{0}\right)} n_{x} \leqslant \rho_{\ell} \lambda_{\Delta} v_{L}(1+\epsilon),
$$

where $\lambda_{\Delta}$ is the largest solution to the equation

$$
4 \Delta \sqrt{\lambda}(1-\lambda)=1
$$

in $[0,1]$. Moreover, all the other external contours $\gamma \neq \gamma_{0}$ satisfy $\operatorname{diam} \gamma \leqslant K \log L$.

Remark 2.2. We note that, in the case $\Delta=\Delta_{\mathrm{c}}$, there is at most one large external contour satisfying the bounds (2.12)-(2.13), or no contour beyond $K \log L$ at all. The details of what exactly happens when $\Delta=\Delta_{\mathrm{c}}$ have not, at present, been quantified - presumably, these will depend on the precise asymptotic of the sequence $v_{L}$.

Remark 2.3. One additional piece of information we could add about the contour $\gamma_{0}$ is that its macroscopic shape asymptotically optimizes the Wulff functional, see (2.6-2.7). While the shape of the unique large contour plays no essential role in this paper (it appears implicitly in the value $w_{1}$ ) we note that statements of this sort were the basis of the (microscopic) Wulff construction, initiated in refs. 1 and 14 for the case of 2D Ising model and percolation. These 2D results were later extended in ref. 15, 19-21, 26, 27. The techniques developed in these papers have been instrumental for the results of ref. 5, which addresses the regime that is "critical" for droplet formation. Recently, extensions going beyond two spatial dimensions have also been accomplished. ${ }^{(6,10,11)}$ We refer to refs. 5 and 7 for more information on the subject.

\subsection{Gibbs-Thomson Formula(s) for 2D Ising Lattice Gas}

Now we are finally in a position to state our rigorous version of the Gibbs-Thomson formula for the 2D Ising lattice gas. We will begin with the formula for the difference of the densities, which is, more or less, an immediate corollary of Theorem B.

Theorem 2.4. Let $\beta>\beta_{\mathrm{c}}$ and let the sequence $\left(v_{L}\right)$ and the quantities $\rho_{\mathrm{g}}=\rho_{\mathrm{g}}(\beta), \rho_{\ell}=\rho_{\ell}(\beta), \varkappa=\varkappa(\beta)$, and $w_{1}=w_{1}(\beta)$ be as defined previously. Let $\Delta \in(0, \infty)$ be as in (2.9). Suppose that $\Delta>\Delta_{\mathrm{c}}=\frac{1}{2}(3 / 2)^{3 / 2}$ and let $\lambda_{\Delta}$ be the largest solution of the equation (2.14) in the interval $[0,1]$. Let $\mathscr{A}_{\epsilon, L}$ be the set of configurations $\left(n_{x}\right)_{x \in A_{L}}$ that contain a unique large 
external contour $\gamma_{0}$-as described in Theorem B - obeying (2.12-2.13), and whose particle density in the exterior of $\gamma_{0}$,

$$
\rho_{\text {ext }}\left(\gamma_{0}\right)=\frac{1}{\left|\Lambda_{L} \backslash V\left(\gamma_{0}\right)\right|} \sum_{x \in \Lambda_{L} \backslash V\left(\gamma_{0}\right)} n_{x}
$$

satisfies the bounds

$$
\begin{aligned}
\frac{1}{2} \frac{x w_{1}}{\rho_{\ell}-\rho_{\mathrm{g}}} \frac{1}{\left|V\left(\gamma_{0}\right)\right|^{1 / 2}}(1-\epsilon) & \leqslant \rho_{\text {ext }}\left(\gamma_{0}\right)-\rho_{\mathrm{g}} \\
& \leqslant \frac{1}{2} \frac{x w_{1}}{\rho_{\ell}-\rho_{\mathrm{g}}} \frac{1}{\left|V\left(\gamma_{0}\right)\right|^{1 / 2}}(1+\epsilon) .
\end{aligned}
$$

Then, for each $\epsilon>0$, we have

$$
\lim _{L \rightarrow \infty} P_{L}^{\circ, \beta, \mu_{\mathrm{t}}}\left(\mathscr{A}_{\epsilon, L}\left|N_{L}=\rho_{\mathrm{g}}\right| \Lambda_{L} \mid+\left(\rho_{\ell}-\rho_{\mathrm{g}}\right) v_{L}\right)=1
$$

Remark 2.5. We note that, up to the $\epsilon$ corrections, (2.16) is exactly (1.5) for $d=2$. Indeed, by Theorem B we know that $\left|V\left(\gamma_{0}\right)\right|=$ $\lambda_{\Delta} v_{L}(1+o(1))$ and the two formulas are identified by noting that $\delta V$ corresponds to $v_{L}$ in our setting. Due to the underlying lattice, the Wulff droplet is undoubtedly not circular for any $\beta>\beta_{\mathrm{c}}$ and the better-known form (1.7) of the (density) Gibbs-Thomson formula does not apply.

In order to state our version of the Gibbs-Thomson formula for the pressure, we will first need to define the pressure "above a curved interface" - not to mention the planar interface. We will closely follow the heuristic definitions $(1.8-1.11)$. Let us consider a sequence $\left(\Lambda_{L}^{\prime}\right)$ of squares in $\mathbb{Z}^{2}$ satisfying

$$
\Lambda_{L}^{\prime} \supset \Lambda_{L} \quad \text { but } \quad \Lambda_{L}^{\prime} \neq \Lambda_{L}
$$

for all $L$. Let $Z_{\mathrm{C}}^{\circ, \beta}(N, \Lambda)$ denote the canonical partition function in $\Lambda$ with $N$ particles, inverse temperature $\beta$ and the vacant boundary condition. This quantity is computed by summing the Boltzmann factor,

$$
\exp \left\{\beta \sum_{\substack{\langle x, y\rangle \\ x, y \in \Lambda}} n_{x} n_{y}\right\},
$$


over all configurations $\left(n_{x}\right)$ with $\sum_{x \in \Lambda} n_{x}=N$. Then we let

$$
p_{L}=\frac{1}{\beta} \frac{1}{\left|\Lambda_{L}^{\prime} \backslash \Lambda_{L}\right|} \log \frac{Z_{\mathrm{C}}^{\circ, \beta}\left(\rho_{\mathrm{g}}\left|\Lambda_{L}\right|+\left(\rho_{\ell}-\rho_{\mathrm{g}}\right) v_{L}, \Lambda_{L}^{\prime}\right)}{Z_{\mathrm{C}}^{\circ, \beta}\left(\rho_{\mathrm{g}}\left|\Lambda_{L}\right|+\left(\rho_{\ell}-\rho_{\mathrm{g}}\right) v_{L}, \Lambda_{L}\right)} .
$$

As in the heuristic section, the quantity $p_{L}$ depends on the sequences $\left(\Lambda_{L}^{\prime}\right),\left(v_{L}\right)$, inverse temperature $\beta$, and also the boundary condition - all of which is notationally suppressed.

For the pressure "above a planar interface," again we will simply use the pressure of the pure (gaseous) phase. If $\Lambda \subset \mathbb{Z}^{2}$ is a finite set, we let $Z_{\mathrm{G}}^{\circ}, \beta(\mu, \Lambda)$ denote the grand canonical partition function in $\Lambda$ corresponding to the chemical potential $\mu$ and vacant boundary condition. Recalling that $\mu_{\mathrm{t}}=2$, we define

$$
p_{\infty}=\frac{1}{\beta} \lim _{L \rightarrow \infty} \frac{1}{\left|\Lambda_{L}\right|} \log Z_{\mathrm{G}}^{\circ, \beta}\left(\mu_{\mathrm{t}}, \Lambda_{L}\right),
$$

where the limit exists by standard subadditivity arguments.

Suppose that $\Delta>\Delta_{\mathrm{c}}$ and let us consider the event $\mathscr{B}_{\epsilon, L}$ collecting all configurations in $\Lambda_{L}$ that have a unique "large" contour $\gamma_{0}$, as described in Theorem B, such that, in addition to (2.12)-(2.13), the volume $V\left(\gamma_{0}\right)$ satisfies the inequalities

$$
\begin{aligned}
\frac{1}{2} \frac{\rho_{\mathrm{g}} w_{1}}{\rho_{\ell}-\rho_{\mathrm{g}}} \frac{1}{\left|V\left(\gamma_{0}\right)\right|^{1 / 2}}(1-\epsilon) & \leqslant \beta\left(p_{L}-p_{\infty}\right) \\
& \leqslant \frac{1}{2} \frac{\rho_{\mathrm{g}} w_{1}}{\rho_{\ell}-\rho_{\mathrm{g}}} \frac{1}{\left|V\left(\gamma_{0}\right)\right|^{1 / 2}}(1+\epsilon) .
\end{aligned}
$$

Somewhat informally, the event $\mathscr{B}_{\epsilon, L}$ represents the configurations for which the Gibbs-Thomson formula for pressure holds up to an $\epsilon$ error. The next theorem shows that, as $L \rightarrow \infty$, these configurations exhaust all of the conditional measure (2.11):

Theorem 2.6. Let $\beta>\beta_{\mathrm{c}}$ and let the sequence $\left(v_{L}\right)$ and the quantities $\rho_{\mathrm{g}}=\rho_{\mathrm{g}}(\beta), \rho_{\ell}=\rho_{\ell}(\beta), \varkappa=\chi(\beta)$, and $w_{1}=w_{1}(\beta)$ be as defined previously. Let $\Delta \in(0, \infty)$ be as in (2.9). Suppose that $\Delta>\Delta_{\mathrm{c}}=\frac{1}{2}(3 / 2)^{3 / 2}$ and let $\lambda_{\Delta}$ be the largest solution to (2.14) in $[0,1]$. For each $\epsilon>0$, there exists a number $\eta_{0}>0$ such that if $\left(\Lambda_{L}^{\prime}\right)$ is a sequence of squares in $\mathbb{Z}^{2}$ satisfying (2.18) and

$$
\lim _{L \rightarrow \infty} \frac{\left|\partial \Lambda_{L}^{\prime}\right|-\left|\partial \Lambda_{L}\right|}{\left|\Lambda_{L}^{\prime} \backslash \Lambda_{L}\right|} \sqrt{v_{L}}=0 \quad \text { and } \quad \lim _{L \rightarrow \infty} \frac{\left|\Lambda_{L}^{\prime} \backslash \Lambda_{L}\right|}{v_{L}}=\eta,
$$


with $\eta \in\left(0, \eta_{0}\right]$, then

$$
\lim _{L \rightarrow \infty} P_{L}^{\circ, \beta, \mu_{\mathrm{t}}}\left(\mathscr{B}_{\epsilon, L}\left|N_{L}=\rho_{\mathrm{g}}\right| \Lambda_{L} \mid+\left(\rho_{\ell}-\rho_{\mathrm{g}}\right) v_{L}\right)=1 .
$$

Remark 2.7. As before, since $\left|V\left(\gamma_{0}\right)\right|=\lambda_{\Delta} v_{L}(1+o(1))$, the equality (2.24) is a rigorous version of (1.21) for the case at hand. The rate at which the limit in (2.24) is achieved depends - among other things - on the rate of the convergence in (2.23). We note that the constraints (2.23) correspond to the bounds in (1.23). In particular, there is a non-trivial set of sequences $\left(\Lambda_{L}^{\prime}\right)$ for which both limits in (2.23) are exactly as prescribed. Finally, the restriction that $\eta>0$ in (2.23) is due to the fact that from ref. 5 we have essentially no control on the rate of convergence in (2.10). Thus, to allow the second limit in (2.23) to be zero, we would have to do a little extra work in order to clarify the rate at which the limits in (2.23) and (2.10) are achieved.

\section{PROOFS OF MAIN RESULTS}

\subsection{Proofs of Theorems 2.4 and 2.6}

In this section we provide the proofs of our main results. We will commence with Theorem 2.4:

Proof of Theorem 2.4. The proof closely follows the heuristic calculation from Section 1.2. Fix an $\epsilon>0$ and let us restrict our attention to particle configurations containing a unique external contour $\gamma_{0}$ and satisfying the bounds (2.12-2.13). Recall the definition (2.8) of the quantity $N_{L}$. We will show that, under the condition

$$
N_{L}=\rho_{\mathrm{g}}\left|\Lambda_{L}\right|+\left(\rho_{\ell}-\rho_{\mathrm{g}}\right) v_{L},
$$

any such configuration is, for a suitable $\epsilon^{\prime}>0$, contained in $\mathscr{A}_{\epsilon^{\prime}, L}$ for all $L$. Introduce the quantity

$$
N_{\text {ext }}\left(\gamma_{0}\right)=\sum_{x \in \Lambda_{L} \backslash V\left(\gamma_{0}\right)} n_{x} .
$$

The inequalities in (2.13) then directly imply

$$
\left|N_{\text {ext }}\left(\gamma_{0}\right)-\left(N_{L}-\rho_{\ell} \lambda_{\Delta} v_{L}\right)\right| \leqslant \epsilon \rho_{\ell} \lambda_{\Delta} v_{L} .
$$

Since we work with a measure conditioned on the event (3.1), we can write

$$
N_{L}-\rho_{\ell} \lambda_{\Delta} v_{L}=\rho_{\mathrm{g}}\left(\left|\Lambda_{L}\right|-\lambda_{\Delta} v_{L}\right)+\left(\rho_{\ell}-\rho_{\mathrm{g}}\right)\left(1-\lambda_{\Delta}\right) v_{L} .
$$


But $\left|\Lambda_{L}\right|-\lambda_{\Delta} v_{L}=\left|\Lambda_{L} \backslash V\left(\gamma_{0}\right)\right|+\left(\left|V\left(\gamma_{0}\right)\right|-\lambda_{\Delta} v_{L}\right)$ and by (2.12), the second term is no larger than $\epsilon \lambda_{\Delta} v_{L}$. Combining the previous estimates, we derive the bound

$$
\left|N_{\text {ext }}\left(\gamma_{0}\right)-\rho_{\mathrm{g}}\right| \Lambda_{L} \backslash V\left(\gamma_{0}\right)\left|-\left(\rho_{\ell}-\rho_{\mathrm{g}}\right)\left(1-\lambda_{\Delta}\right) v_{L}\right| \leqslant \epsilon \lambda_{\Delta} v_{L},
$$

where we also used (inessentially) that $\rho_{\ell}+\rho_{\mathrm{g}}=1$.

The first two terms in the absolute value on the left-hand side represent the difference between $\rho_{\text {ext }}\left(\gamma_{0}\right)$ and $\rho_{\mathrm{g}}$ while the third term is exactly the Gibbs-Thomson correction. Indeed, dividing (3.5) by $\left|\Lambda_{L} \backslash V\left(\gamma_{0}\right)\right|$ and noting that, by definition, $N_{\text {ext }}\left(\gamma_{0}\right)=\rho_{\text {ext }}\left(\gamma_{0}\right)\left|\Lambda_{L} \backslash V\left(\gamma_{0}\right)\right|$, we get

$$
\left|\rho_{\text {ext }}\left(\gamma_{0}\right)-\rho_{\mathrm{g}}-\left(\rho_{\ell}-\rho_{\mathrm{g}}\right) \frac{\left(1-\lambda_{\Delta}\right) v_{L}}{\left|\Lambda_{L} \backslash V\left(\gamma_{0}\right)\right|}\right| \leqslant \frac{\epsilon \lambda_{\Delta} v_{L}}{\left|\Lambda_{L} \backslash V\left(\gamma_{0}\right)\right|} .
$$

Since both the Gibbs-Thomson correction - which arises from the last term in the above absolute value - and the error term on the right-hand side are proportional to $v_{L} /\left|\Lambda_{L} \backslash V\left(\gamma_{0}\right)\right|$, the desired bound (2.16) will follow with some $\epsilon>0$ once we show that

$$
\left(\rho_{\ell}-\rho_{\mathrm{g}}\right) \frac{\left(1-\lambda_{\Delta}\right) v_{L}}{\left|\Lambda_{L} \backslash V\left(\gamma_{0}\right)\right|}=\frac{1}{2} \frac{\chi w_{1}}{\rho_{\ell}-\rho_{\mathrm{g}}} \frac{1}{\sqrt{\lambda_{\Delta} v_{L}}}(1+o(1)), \quad L \rightarrow \infty .
$$

To prove (3.7), we note that $\left|\Lambda_{L} \backslash V\left(\gamma_{0}\right)\right| /\left|\Lambda_{L}\right|=1+o(1)$, which using (2.9) allows us to write

$$
\frac{v_{L}}{\left|\Lambda_{L} \backslash V\left(\gamma_{0}\right)\right|}=\frac{2 \varkappa w_{1}}{\left(\rho_{\ell}-\rho_{\mathrm{g}}\right)^{2}} \frac{\Delta}{\sqrt{v_{L}}}(1+o(1)), \quad L \rightarrow \infty .
$$

Using (2.14) in the form $\Delta\left(1-\lambda_{\Delta}\right)=1 /\left(4 \sqrt{\lambda_{\Delta}}\right)$, we get rid of the factor of $\Delta$, whereby (3.7) follows. Since the $o(1)$ term in (3.7) is uniformly small for all configurations satisfying (2.12-2.13), the bounds (2.16) hold once $L$ is sufficiently large.

In order to prove our Gibbs-Thomson formula for the pressure, we will need the following representation of the grand canonical partition function:

Theorem 3.1. Let $\beta>\beta_{\mathrm{c}}$ and let $p_{\infty}$ be as in (2.21). There exists a number $\tau_{\text {wall }}^{\circ} \in \mathbb{R}$ and, for each $\theta \in(1, \infty)$, also a constant $C(\beta, \theta)<\infty$ such that

$$
\left|\log Z_{\mathrm{G}}^{\circ, \beta}\left(\mu_{\mathrm{t}}, \Lambda\right)-\beta p_{\infty}\right| \Lambda\left|-\tau_{\text {wall }}^{\circ}\right| \partial \Lambda|| \leqslant C(\beta, \theta)
$$


holds for all rectangular volumes $\Lambda \subset \mathbb{Z}^{2}$ whose aspect ratio lies in the interval $\left(\theta^{-1}, \theta\right)$.

Clearly, Theorem 3.1 is a rigorous version of the formula (1.10). Such things are well known in the context of low-temperature expansions, see, e.g., ref. 8. Here we are using expansion techniques in conjunction with correlation inequalities to get the claim "down to $\beta_{\mathrm{c}}$." However, the full argument would detract from the main line of thought, so the proof is postponed to Section 3.2.

Proof of Theorem 2.6. We will again closely follow the heuristic derivation from Section 1.2. First we note that, using Theorem 3.1, we have

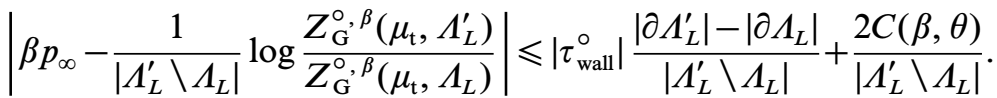

Introducing the shorthand

$$
\mathbb{P}_{\Lambda}(N)=P_{\Lambda}^{\circ, \beta, \mu_{t}}\left(\sum_{x \in \Lambda} n_{x}=N\right),
$$

invoking the assumption on the left of (2.23) and applying (2.20), this allows us to write

$\beta\left(p_{L}-p_{\infty}\right)=\frac{1}{\left|\Lambda_{L}^{\prime} \backslash \Lambda_{L}\right|} \log \frac{\mathbb{P}_{\Lambda_{L}}\left(\rho_{\mathrm{g}}\left|\Lambda_{L}\right|+\left(\rho_{\ell}-\rho_{\mathrm{g}}\right) v_{L}\right)}{\mathbb{P}_{\Lambda_{L}}\left(\rho_{\mathrm{g}}\left|\Lambda_{L}\right|+\left(\rho_{\ell}-\rho_{\mathrm{g}}\right) v_{L}\right)}+o\left(v_{L}^{-1 / 2}\right)$,

as $L \rightarrow \infty$. Now, by Theorem A we have

$$
\log \mathbb{P}_{\Lambda_{L}}\left(\rho_{\mathrm{g}}\left|\Lambda_{L}\right|+\left(\rho_{\ell}-\rho_{\mathrm{g}}\right) v_{L}\right)=-w_{1}\left(\Phi_{\Delta}^{\star}+o(1)\right) \sqrt{v_{L}}, \quad L \rightarrow \infty,
$$

where $\Phi_{\Delta}^{\star}$ is the absolute minimum of $\Phi_{\Delta}(\lambda)$ for $\lambda \in[0,1]$. As to the corresponding probability for $\Lambda_{L}^{\prime}$, we first note that

$$
\rho_{\mathrm{g}}\left|\Lambda_{L}\right|+\left(\rho_{\ell}-\rho_{\mathrm{g}}\right) v_{L}=\rho_{\mathrm{g}}\left|\Lambda_{L}^{\prime}\right|+\left(\rho_{\ell}-\rho_{\mathrm{g}}\right) \alpha_{L} v_{L},
$$

where

$$
\alpha_{L}=1-\frac{\rho_{\mathrm{g}}}{\rho_{\ell}-\rho_{\mathrm{g}}} \frac{\left|\Lambda_{L}^{\prime} \backslash \Lambda_{L}\right|}{v_{L}} .
$$

By our assumption on the right-hand side of (2.23), $\alpha_{L}$ converges to a number $\alpha$ given by $\alpha=1-\frac{\rho_{\mathrm{g}}}{\rho_{\ell}-\rho_{\mathrm{g}}} \eta$. Again using Theorem A, we can write

$$
\log \mathbb{P}_{\Lambda_{L}}\left(\rho_{\mathrm{g}}\left|\Lambda_{L}\right|+\left(\rho_{\ell}-\rho_{\mathrm{g}}\right) v_{L}\right)=-w_{1}\left(\Phi_{\alpha^{3 / 2} \Delta}^{\star}+o(1)\right) \sqrt{\alpha} \sqrt{v_{L}},
$$


as $L \rightarrow \infty$. A simple calculation-of the kind leading to (1.20)-now shows that

$$
\sqrt{\alpha} \Phi_{\alpha^{3 / 2} \Delta}^{\star}-\Phi_{\Delta}^{\star}=\frac{\eta}{2} \frac{\rho_{\mathrm{g}}}{\rho_{\ell}-\rho_{\mathrm{g}}} \frac{1}{\sqrt{\lambda_{\Delta}}}+O\left(\eta^{2}\right), \quad \eta \downarrow 0,
$$

while (2.23) implies that

$$
\frac{\sqrt{v_{L}}}{\left|\Lambda_{L}^{\prime} \backslash \Lambda_{L}\right|}=\frac{1}{\sqrt{v_{L}}} \frac{1}{\eta}(1+o(1)), \quad L \rightarrow \infty .
$$

Plugging these equations, along with (3.13) and (3.16), into (3.12), we have

$$
\beta\left(p_{L}-p_{\infty}\right)=\frac{1}{2} \frac{\rho_{\mathrm{g}} w_{1}}{\rho_{\ell}-\rho_{\mathrm{g}}} \frac{1}{\sqrt{\lambda_{\Delta} v_{L}}}\left(1+\frac{o(1)}{\eta}+O(\eta)\right),
$$

where $o(1)$ denotes a quantity tending to zero as $L \rightarrow \infty$ while $O(\eta)$ is a quantity independent of $L$ and tending to zero at least as fast as $\eta$ in the limit $\eta \downarrow 0$. Equation (3.19) shows that, once $L$ is sufficiently large, a particle configuration satisfying the bounds (2.12) from Theorem B will also satisfy the bounds (2.22). The limit (2.24) is then a simple conclusion of Theorem B.

\subsection{Representation of the Partition Function}

The goal of this section is to prove Theorem 3.1. As already mentioned, we will employ two basic techniques: cluster expansion and correlation inequalities. The basic strategy of the proof is as follows. First we pick a large negative number $\mu_{0}<\mu_{\mathrm{t}}$ and use cluster expansion to establish a corresponding representation for the partition function $Z_{\mathrm{G}}^{\circ}, \beta\left(\mu_{0}, \Lambda_{L}\right)$. Then, as a second step, we invoke correlation inequalities to prove a similar representation for the ratio of the partition functions $Z_{\mathrm{G}}^{\circ, \beta}\left(\mu_{0}, \Lambda_{L}\right)$ and $Z_{\mathrm{G}}^{\circ}, \beta\left(\mu_{\mathrm{t}}, \Lambda_{L}\right)$. Essential for the second step will be the GHS inequality and the exponential decay of correlations for all $\beta>\beta_{\mathrm{c}}$. Combining these two steps, the desired representation will be proved.

Let $p_{\infty}(\mu)$ denote the pressure corresponding to the chemical potential $\mu$, which is defined by the limit as in (2.21) where $\mu_{\mathrm{t}}$ is replaced by $\mu$. (Throughout this derivation, we will keep $\beta$ fixed and suppress it notationally whenever possible.) The first step in the above strategy can then be formulated as follows: 
Lemma 3.2. Let $\beta>\beta_{\mathrm{c}}$ and let $p_{\infty}(\mu)$ be as defined above. For each $\theta \in(1, \infty)$ and each sufficiently large negative $\mu_{0}$, there exists a number $\tau_{1}^{\circ}\left(\mu_{0}\right) \in \mathbb{R}$ and a constant $C_{1}\left(\beta, \mu_{0}, \theta\right)<\infty$ such that

$$
\left|\log Z_{\mathrm{G}}^{\circ}, \beta\left(\mu_{0}, \Lambda\right)-\beta p_{\infty}\left(\mu_{0}\right)\right| \Lambda\left|-\tau_{1}^{\circ}\left(\mu_{0}\right)\right| \partial \Lambda|| \leqslant C_{1}\left(\beta, \mu_{0}, \theta\right)
$$

holds for each rectangular volume $\Lambda \subset \mathbb{Z}^{2}$ whose aspect ratio lies in the interval $\left(\theta^{-1}, \theta\right)$.

To implement the second step of the proof, we need to study the ratio of the partition functions with chemical potentials $\mu_{\mathrm{t}}$ and $\mu_{0}$. Let $\Lambda$ be a finite rectangular volume in $\mathbb{Z}^{2}$ and let $\langle-\rangle_{A}^{\circ}, \beta, \mu$ denote the expectation with respect to the measure in (2.2) with vacant boundary condition. Let $N_{\Lambda}=\sum_{x \in \Lambda} n_{x}$. For any $\mu_{0}<\mu_{\mathrm{t}}$ we then have

$$
\log \frac{Z_{\mathrm{G}}^{\circ, \beta}\left(\mu_{\mathrm{t}}, \Lambda_{L}\right)}{Z_{\mathrm{G}}^{\circ, \beta}\left(\mu_{0}, \Lambda_{L}\right)}=\int_{\mu_{0}}^{\mu_{\mathrm{t}}}\left\langle N_{\Lambda}\right\rangle_{\Lambda}^{\circ, \beta, \mu} \mathrm{d} \mu
$$

and

$$
\beta\left(p_{\infty}\left(\mu_{\mathrm{t}}\right)-p_{\infty}\left(\mu_{0}\right)\right)=\int_{\mu_{0}}^{\mu_{\mathrm{t}}}\left\langle n_{0}\right\rangle^{\circ, \beta, \mu} \mathrm{d} \mu
$$

where $\langle-\rangle^{\circ, \beta, \mu}$ denotes the infinite-volume limit (which we are assured exists) of the state $\langle-\rangle_{A}^{\circ}, \beta, \mu$. (Note that (3.22) is true with any infinitevolume Gibbs state substituted.) Combining (3.21)-(3.22), we thus get

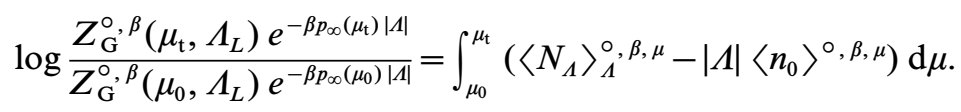

To derive the desired representation, we need to show that the integrand is proportional to $|\partial \Lambda|$, up to an error which does not depend on $\Lambda$. This estimate is provided in the following lemma:

Lemma 3.3. Let $\beta>\beta_{\mathrm{c}}$ and $\theta \in(1, \infty)$. There exists a constant $C_{2}(\beta, \theta)<\infty$ and a bounded function $\tau_{2}^{\circ}:\left(-\infty, \mu_{\mathrm{t}}\right] \rightarrow \mathbb{R}$ such that

$$
\left|\left\langle N_{\Lambda}\right\rangle_{\Lambda}^{\circ, \beta, \mu}-\right| \Lambda\left|\left\langle n_{0}\right\rangle^{\circ, \beta, \mu}-\right| \partial \Lambda\left|\tau_{2}^{\circ}(\mu)\right| \leqslant C_{2}(\beta, \theta),
$$

holds for each $\mu \in\left(-\infty, \mu_{\mathrm{t}}\right]$ and each rectangular volume $\Lambda \subset \mathbb{Z}^{2}$ whose aspect ratio lies in the interval $\left(\theta^{-1}, \theta\right)$.

Lemma 3.2 will be proved in Section 3.3 and Lemma 3.3 in Section 3.4. With the two lemmas in the hand, the proof of Theorem 3.1 is easily concluded: 
Proof of Theorem 3.1. Let $\theta \in(1, \infty)$ and let $\Lambda$ be a rectangular volume whose aspect ratio lies in the interval $\left(\theta^{-1}, \theta\right)$. Fix $\mu_{0}$ to be so large (and negative) that Lemma 3.2 holds and let $Q_{1}\left(\mu_{0}\right)$ denote the quantity in the absolute value in (3.20). For each $\mu \in\left[\mu_{0}, \mu_{\mathrm{t}}\right]$, let $Q_{2}(\mu)$ denote the quantity inside the absolute value in (3.24). Let us define

$$
\tau_{\text {wall }}^{\circ}=\tau_{1}^{\circ}\left(\mu_{0}\right)+\int_{\mu_{0}}^{\mu_{\mathrm{t}}} \tau_{2}^{\circ}(\mu) \mathrm{d} \mu .
$$

A simple calculation combining (3.20), (3.24), with (3.23) then shows that

$$
\log Z_{\mathrm{G}}^{\circ, \beta}(\mu, \Lambda)-\beta p_{\infty}\left(\mu_{\mathrm{t}}\right)|\Lambda|-\tau_{\text {wall }}^{\circ}|\partial \Lambda|=Q_{1}\left(\mu_{0}\right)+\int_{\mu_{0}}^{\mu_{\mathrm{t}}} Q_{2}(\mu) \mathrm{d} \mu .
$$

Using (3.20) and (3.24), we easily establish that the absolute value of the quantity on right-hand side is no larger than $C(\beta, \theta)=C_{1}\left(\beta, \mu_{0}, \theta\right)+$ $\left(\mu_{\mathrm{t}}-\mu_{0}\right) C_{2}(\beta, \theta)$.

\subsection{Cluster Expansion}

Here we will rewrite the grand canonical partition function in terms of a polymer model, then we will collect a few facts from the theory of cluster expansions and assemble them into the proof of Lemma 3.2. The substance of this section is very standard-mostly siphoned from ref. 23-so the uninterested reader may wish to consider skipping the entire section on a first reading.

We begin by defining the polymer model. Given a configuration $n_{A}$ in $\Lambda$, let us call two distinct sites of $\mathbb{Z}^{2}$ connected if they are nearest-neighbors and are both occupied in the configuration $n_{\Lambda}$. A polymer is then defined as a connected component of occupied sites. Two polymers are called compatible if their union is not connected. A collection of polymers is called compatible if each distinct pair of polymers within the collection is compatible. Clearly, the compatible collections of polymers are in one-to-one correspondence with the particle configurations. Finally, let us introduce some notation: We write $\mathrm{P} \times \mathrm{P}^{\prime}$ if the polymers $\mathrm{P}$ and $\mathrm{P}^{\prime}$ are not compatible and say that the polymer $\mathrm{P}$ is in $\Lambda$ if $\mathrm{P} \subset \Lambda$.

Let $\mathrm{P}$ be a polymer containing $N(\mathrm{P})$ sites and occupying both endpoints of $E(\mathrm{P})$ edges in $\mathbb{Z}^{2}$. We define the Boltzmann weight of $\mathrm{P}$ by the formula

$$
\zeta_{\beta, \mu}(\mathrm{P})=e^{\beta E(\mathrm{P})+\mu N(\mathrm{P})} .
$$


As is straightforward to verify, the partition function $Z_{\mathrm{G}}^{\circ, \beta}(\mu, \Lambda)$ can be written as

$$
Z_{\mathrm{G}}^{\circ, \beta}(\mu, \Lambda)=\sum_{\mathscr{P}} \prod_{\mathrm{P} \in \mathscr{P}} \zeta_{\beta, \mu}(\mathrm{P}),
$$

where the sum runs over all compatible collections $\mathscr{P}$ of polymers in $\Lambda$.

This reformulation of the partition function in the language of compatible polymer configurations allows us to bring to bear the machinery of cluster expansion. Following, ${ }^{(23)}$ the next key step is a definition of a cluster, generically denoted by $\mathrm{C}$, by which we will mean a finite non-empty collection of polymers that is connected when viewed as a graph with vertices labeled by polymers $\mathrm{P} \in \mathrm{C}$ and edges connecting pairs of incompatible polymers. (Thus, if $\mathrm{C}$ contains but a single polymer it is automatically a cluster. If $\mathrm{C}$ contains more than one polymer, then any non-trivial division of $\mathrm{C}$ into two disjoint subsets has some incompatibility between some pair chosen one from each of the subsets.) In accord with ref. 23, a cluster $C$ is incompatible with a polymer $P$, expressed by $C \nsim P$, if $C \cup\{P\}$ is a cluster.

In order to use this expansion, we need to verify the convergence criterion from ref. 23. In present context this reads as follows: For some $\kappa \geqslant 0$ and any polymer $\mathrm{P}$,

$$
\sum_{\mathrm{P}^{\prime}: \mathrm{P}^{\prime} \neq \mathrm{P}} \zeta_{\beta, \mu}\left(\mathrm{P}^{\prime}\right) e^{(1+\kappa) N\left(\mathrm{P}^{\prime}\right)} \leqslant N(\mathrm{P})
$$

Since $\zeta_{\beta, \mu}(\mathrm{P}) \leqslant e^{(\mu+2 \beta) N(\mathrm{P})}$ is true, this obviously holds if $\mu$ is sufficiently large and negative. The main result of ref. 23 then says that each cluster $C$ can be given a weight $\zeta_{\beta, \mu}$ (C) (which is defined less implicitly in ref. 23), such that for all finite volumes $\Lambda \subset \mathbb{Z}^{2}$ we have

$$
\log Z_{\mathrm{G}}^{\circ, \beta}(\mu, \Lambda)=\sum_{\mathrm{C} \in \mathscr{C}_{\Lambda}} \zeta_{\beta, \mu}(\mathrm{C}),
$$

where $\mathscr{C}_{\Lambda}$ denotes the set of all clusters arising from polymers in $\Lambda$. Moreover, this expansion is accompanied by the bound

$$
\sum_{\mathrm{C}: \mathrm{C} \nsim \mathrm{P}}\left|\zeta_{\beta, \mu}(\mathrm{C})\right| e^{\kappa N(\mathrm{C})} \leqslant N(\mathrm{P})
$$

where $N(\mathrm{C})$ denotes the sum of $N\left(\mathrm{P}^{\prime}\right)$ over all $\mathrm{P}^{\prime}$ constituting $\mathrm{C}$. With (3.30)-(3.31) in hand, we are now ready to prove the first part of the representation of $Z_{\mathrm{G}}^{\circ}, \beta(\mu, \Lambda)$ : 
Proof of Lemma 3.2. First, we will introduce a convenient resummation of (3.30). For each polymer $\mathrm{P}$, let $\mathscr{N}(\mathrm{P})$ be the set of sites constituting $\mathrm{P}$. Similarly, for each cluster $\mathrm{C}$, let $\mathscr{N}(\mathrm{C})$ be the union of $\mathscr{N}(\mathrm{P})$ over all $\mathrm{P}$ constituting $\mathrm{C}$. For each finite $A \subset \mathbb{Z}^{2}$, we let

$$
\vartheta_{\beta, \mu}(A)=\sum_{\mathrm{C}: \mathcal{N}(\mathrm{C})=A} \zeta_{\beta, \mu}(C)
$$

Clearly, the weights $\vartheta_{\beta, \mu}$ are invariant with respect to lattice translations and rotations, having inherited this property from $\zeta_{\beta, \mu}$. Moreover, as is easily checked, $\vartheta_{\beta, \mu}(A)=0$ unless $A$ is a connected set. The new weights allow us to rewrite (3.30) and (3.31) in the following form:

$$
\log Z_{\mathrm{G}}^{\circ, \beta}(\mu, \Lambda)=\sum_{A: A \subset \Lambda} \vartheta_{\beta, \mu}(A)
$$

with

$$
\sum_{\substack{A: 0 \in A \\|A| \geqslant n}}\left|\vartheta_{\beta, \mu}(A)\right| \leqslant e^{-\kappa n}
$$

for each $n \geqslant 0$. Here $|A|$ denotes the number of sites in $A$.

Now we are in a position to identify the relevant quantities. First, the limiting version of the expression (3.33) suggests that the pressure should be given by the formula

$$
\beta p_{\infty}(\mu)=\sum_{A: 0 \in A} \frac{1}{|A|} \vartheta_{\beta, \mu}(A)
$$

To define the constant $\tau_{1}^{\circ}(\mu)$ representing the wall surface tension, let $\mathbb{W}$ denote the upper half-plane in $\mathbb{Z}^{2}$, i.e., $\mathbb{H}=\left\{\left(x_{1}, x_{2}\right) \in \mathbb{Z}^{2}: x_{2}>0\right\}$, and let $\mathbb{L}$ be the "line" in $\mathbb{Z}^{2}$ corresponding to the boundary of $\mathbb{R}$, i.e., $\mathbb{L}=$ $\left\{\left(x_{1}, x_{2}\right) \in \mathbb{Z}^{2}: x_{2}=0\right\}$. Then we define

$$
\tau_{1}^{\circ}(\mu)=-\sum_{\substack{A: 0 \in A \\ A \cap \mathbb{L} \neq \varnothing}} \frac{|A \cap \mathbb{H}|}{|A|} \frac{\vartheta_{\beta, \mu}(A)}{|A \cap \mathbb{\mathbb { L }}|} .
$$

Clearly, in order to contribute to $\tau_{1}^{\circ}(\mu)$, the set $A$ would have to have both $A \cap \mathbb{W}$ and $A \backslash \mathbb{W}$ nonempty. On the basis of (3.34) it can be shown that the sums in (3.35) and (3.36) converge once (3.29) holds with a $\kappa>0$. 
Combining (3.33) with (3.35), we can now write that

$$
\begin{aligned}
\log Z_{\mathrm{G}}^{\circ, \beta}(\mu, \Lambda) & =\sum_{x \in \Lambda} \sum_{\substack{A: x \in A \\
A \subset A}} \frac{1}{|A|} \vartheta_{\beta, \mu}(A) \\
& =\beta p_{\infty}(\mu)|\Lambda|-\sum_{x \in \Lambda} \sum_{\substack{A: x \in A \\
A \notin A}} \frac{1}{|A|} \vartheta_{\beta, \mu}(A) .
\end{aligned}
$$

Using the fact that $A$ is a connected set and thus $A \cap \Lambda \neq \varnothing$ and $A \backslash \Lambda \neq \varnothing$ imply that $A \cap \partial \Lambda \neq \varnothing$, the second term on the right-hand side can further be written as

$$
\begin{aligned}
& -\sum_{A: A \nsubseteq \Lambda} \frac{|A \cap \Lambda|}{|A|} \vartheta_{\beta, \mu}(A) \\
& =-\sum_{x \in \partial \Lambda} \sum_{A: x \in A} \frac{|A \cap \Lambda|}{|A|} \frac{\vartheta_{\beta, \mu}(A)}{|A \cap \partial \Lambda|} \\
& \quad=\tau_{1}^{\circ}(\mu)|\partial \Lambda|+\sum_{x \in \partial \Lambda} \sum_{A: x \in A} \frac{1}{|A|}\left(\frac{\left|A \cap \mathbb{U}_{x}\right|}{\left|A \cap \mathbb{L}_{x}\right|}-\frac{|A \cap \Lambda|}{|A \cap \partial \Lambda|}\right) \vartheta_{\beta, \mu}(A) .
\end{aligned}
$$

Here $\mathbb{H}_{x}$ denotes the half-plane in $\mathbb{Z}^{2}$ that contains $\Lambda$ and whose boundary $\mathbb{L}_{x}=\partial \mathbb{W}_{x}$ includes the portion of the boundary $\partial \Lambda$ that contains $x$. (Remember that $\Lambda$ is a rectangular set and thus its boundary $\partial \Lambda$ splits into four disjoint subsets - the sides of $\Lambda$.)

Let $Q_{1}(\mu)$ denote the (complicated) second term on the right-hand side of (3.38). Let $\mathscr{A}$ be the collection of all finite connected sets $A \subset \mathbb{Z}^{2}$. Notice that, whenever a set $A \in \mathscr{A}$ intersects $\partial \Lambda$ in only one of its sides and

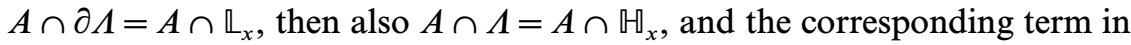
(3.38) vanishes. It follows that, in order for the set $A$ to contribute to the $x$ th term of $Q_{1}(\mu)$, it must contain at least as many sites as is the $\ell^{\infty}$-distance from $x$ to the sides of $\partial \Lambda$ not containing $x$. Thus, for a given $x \in \partial \Lambda$, a set $A \subset \mathbb{Z}^{2}$ can only contribute to $Q_{1}(\mu)$ if $A \in \mathscr{A}$ and $|A| \geqslant$ $\operatorname{dist}\left(x, \partial \Lambda \backslash \mathbb{E}_{x}\right)$.

Since $|A \cap \Lambda|,\left|A \cap \mathbb{H}_{x}\right| \leqslant|A|$ and $|A \cap \partial \Lambda|,\left|A \cap \mathbb{L}_{x}\right| \geqslant 1$ for any $A$ contributing to $Q_{1}(\mu)$, we can use (3.34) to get the bound

$$
\left|Q_{1}(\mu)\right| \leqslant \sum_{x \in \partial A} \sum_{\substack{A \in \mathcal{A}, x \in A \\|A| \geqslant \operatorname{dist}\left(x, \partial \Lambda \backslash \mathbb{L}_{x}\right)}}\left|\vartheta_{\beta, \mu}(A)\right| \leqslant \sum_{x \in \partial \Lambda} e^{-\kappa \operatorname{dist}\left(x, \partial \Lambda \backslash \mathbb{E}_{x}\right)}
$$


Choosing $\kappa>0$, letting $G(\kappa)=\sum_{n=1}^{\infty} e^{-\kappa n}<\infty$, and using $L_{1}, L_{2} \in$ $\left[\theta^{-1} L, \theta L\right]$ to denote the lengths of the sides of $\partial \Lambda$, we can bound the right hand side by $8 G(\kappa)+2 L_{1} e^{-\kappa L_{2}}+2 L_{2} e^{-\kappa L_{1}}$, yielding $\left|Q_{1}(\mu)\right| \leqslant 8 G(\kappa)+$ $4 \theta L e^{-\frac{\kappa}{\theta} L}$. This in turn can be bounded uniformly in $L$ by a constant that depends only on $\theta$ and we thus get the claim of Lemma 3.2.

\subsection{Correlation Bounds}

This section will be spent on proving Lemma 3.3. We begin by recalling the relevant correlation bounds. Let us extend our notation $\langle-\rangle_{A}^{\circ}, \beta, \mu$ for the expectation with respect to the Gibbs measure in $\Lambda$ also to the cases when $\Lambda$ is not necessarily finite. (It turns out that, by FKG monotonicity, such a state is uniquely defined as a limit of finite-volume Gibbs states along any sequence of finite volumes increasing to 4 .) We will use the notation

$$
\left\langle n_{x} ; n_{y}\right\rangle_{A}^{\circ, \beta, \mu}=\left\langle n_{x} n_{y}\right\rangle_{A}^{\circ, \beta, \mu}-\left\langle n_{x}\right\rangle_{A}^{\circ}, \beta, \mu\left\langle n_{y}\right\rangle_{A}^{\circ, \beta, \mu}
$$

for the truncated correlation function. This correlation function has the following properties:

(1) For each $\mu<\mu^{\prime} \leqslant \mu_{\mathrm{t}}$ and $\Lambda \subset \Lambda^{\prime}$, and all $x, y \in \mathbb{Z}^{2}$,

$$
\left\langle n_{x} ; n_{y}\right\rangle_{A}^{\circ, \beta, \mu} \leqslant\left\langle n_{x} ; n_{y}\right\rangle_{A^{\prime}}^{\circ}, \beta, \mu^{\prime} .
$$

(2) For each $\beta>\beta_{\mathrm{c}}$ there exists a $\xi=\xi(\beta)<\infty$ such that

$$
0 \leqslant\left\langle n_{x} ; n_{y}\right\rangle_{A}^{\circ, \beta, \mu} \leqslant e^{-|x-y| / \xi}
$$

for all $\mu \leqslant \mu_{\mathrm{t}}$, all $\Lambda \subset \mathbb{Z}^{2}$ and all $x, y \in \mathbb{Z}^{2}$. Here $|x-y|$ denotes the $\ell_{\infty}$ distance between $x$ and $y$.

Both (1) and (2) are reformulations of well-known properties of the truncated correlation functions for Ising spins. Namely, (1) is a simple consequence of the GHS inequality, ${ }^{(18)}$ while (2) is a consequence of (1) and the fact that the infinite-volume truncated correlation function at $\mu=\mu_{\mathrm{t}}$ decays exponentially once $\beta>\beta_{\mathrm{c}}$. The latter was in turn proved in refs. 12 and 21 . lemma:

A simple consequence of the above observations is the following

Lemma 3.4. Let $\beta>\beta_{\mathrm{c}}$. Then there exist constants $\alpha_{1}=\alpha_{1}(\beta) \in$ $(0, \infty)$ and $\alpha_{2}=\alpha_{2}(\beta) \in(0, \infty)$ such that

$$
0 \leqslant\left\langle n_{x}\right\rangle_{\Lambda^{\prime}}^{\circ}, \beta, \mu-\left\langle n_{x}\right\rangle_{\Lambda}^{\circ, \beta, \mu} \leqslant \alpha_{1} e^{-\alpha_{2} \operatorname{dist}\left(x, \Lambda^{\prime} \backslash \Lambda\right)}
$$


holds for all $\mu \leqslant \mu_{\mathrm{t}}$, all (not necessarily finite) volumes $\Lambda \subset \Lambda^{\prime} \subset \mathbb{Z}^{2}$ and all $x \in \Lambda$.

Proof. See, e.g., formula (2.2.6) from ref. 21; the original derivation goes back to ref. 9 .

Now we can start proving Lemma 3.3:

Proof of Lemma 3.3. We begin by a definition of the quantity $\tau_{2}^{\circ}(\mu)$. Let $\mathbb{W}$ be the upper half-plane in $\mathbb{Z}^{2}$, see Section 3.3. Then we define

$$
\tau_{2}^{\circ}(\mu)=\sum_{\ell \geqslant 1}\left(\left\langle n_{(0, \ell)}\right\rangle_{\mathbb{H}}^{\circ, \beta, \mu}-\left\langle n_{0}\right\rangle_{\mathbb{Z}^{\beta}}^{\circ \beta, \mu}\right),
$$

where $\left(x_{1}, x_{2}\right)$ is a notation for a generic point in $\mathbb{Z}^{2}$. By Lemma 3.4, the sum converges with a $\mu$-independent rate (of course, provided $\mu \leqslant \mu_{\mathrm{t}}$ ).

Let $\Lambda$ be a rectangular volume in $\mathbb{Z}^{2}$ with aspect ratio in the interval $\left(\theta^{-1}, \theta\right)$. Let us cyclically label the sides of $\Lambda$ by numbers $1, \ldots, 4$, and define $\mathbb{H}_{1}, \ldots, \mathbb{W}_{4}$ to be the half-planes in $\mathbb{Z}^{2}$ containing $\Lambda$ and sharing the respective part of the boundary with $\Lambda$. Let us partition the sites of $\Lambda$ into four sets $\Lambda_{1}, \ldots, \Lambda_{4}$ according to which $\mathbb{H}_{j}$ the site is closest to. We resolve the cases of a tie by choosing the $\mathbb{H}_{j}$ with the lowest $j$. Now we can write

$$
\begin{aligned}
\left\langle N_{\Lambda}\right\rangle_{\Lambda}^{\circ, \beta, \mu}-|\Lambda|\left\langle n_{0}\right\rangle_{\mathbb{Z}^{2}}^{\circ, \mu}= & \sum_{j=1}^{4} \sum_{x \in \Lambda_{j}}\left(\left\langle n_{x}\right\rangle_{\Lambda}^{\circ, \beta, \mu}-\left\langle n_{x}\right\rangle_{\mathbb{H}_{j}, \beta, \mu}^{\circ}\right) \\
& +\sum_{j=1}^{4} \sum_{x \in \Lambda_{j}}\left(\left\langle n_{x}\right\rangle_{\mathbb{H}_{j}}^{\circ, \mu, \mu}-\left\langle n_{0}\right\rangle_{\mathbb{Z}^{2}, \beta, \mu}^{\circ}\right) .
\end{aligned}
$$

If it were not for the restriction $x \in \Lambda$, the second term on the right-hand side would have the structure needed to apply (3.44). To fix this problem, let $\mathbb{S}_{j}$, with $j=1, \ldots, 4$, denote the half-infinite slab obtained as the intersection $\mathbb{H}_{j-1} \cap \mathbb{H}_{j} \cap \mathbb{H}_{j+1}$, where it is understood that $\mathbb{W}_{0}=\mathbb{H}_{4}$ and $\mathbb{H}_{5}=\mathbb{M}_{1}$. Clearly, $\Lambda_{j} \subset \mathbb{S}_{j}$ for all $j=1, \ldots, 4$. Then we have

$$
\begin{aligned}
& \sum_{j=1}^{4} \sum_{x \in \Lambda_{j}}\left(\left\langle n_{x}\right\rangle_{\mathbb{H}_{j}^{\prime}, \beta, \mu}^{\circ}-\left\langle n_{0}\right\rangle_{\mathbb{Z}^{2}}^{\circ, \beta, \mu}\right) \\
& \quad=\tau_{2}^{\circ}(\mu)|\partial \Lambda|-\sum_{j=1}^{4} \sum_{x \in \mathbb{S}_{j}(\Lambda) \backslash \Lambda_{j}}\left(\left\langle n_{x}\right\rangle_{\mathbb{H}_{j}}^{\circ, \beta, \mu}-\left\langle n_{0}\right\rangle_{\mathbb{Z}^{2}}^{\circ, \mu, \mu}\right) .
\end{aligned}
$$

It remains to show that both the first term on the right-hand side of (3.45) and the second term on the right-hand side of (3.46) are bounded by 
a constant independent of $\mu$ and $\Lambda$ with the above properties. As to the first term, we note that, by Lemma 3.4,

$$
\left|\left\langle n_{x}\right\rangle_{\Lambda}^{\circ, \beta, \mu}-\left\langle n_{x}\right\rangle_{\mathbb{H}_{j}}^{\circ, \beta, \mu}\right| \leqslant \alpha_{1} e^{-\alpha_{2} \operatorname{dist}\left(x, \mathbb{H}_{j} \backslash \Lambda\right)},
$$

which after summing over $x \in \Lambda_{j}$ gives a plain constant. Concerning the second contribution to the error, we note that $\left\langle n_{x}\right\rangle_{\mathbb{H}_{j}}^{\circ, \beta, \mu}-\left\langle n_{0}\right\rangle_{\mathbb{Z}^{2}}^{\circ, \beta, \mu}$ is again exponentially small in $\operatorname{dist}\left(x, \mathbb{Z}^{2} \backslash \mathbb{H}_{j}\right)$. As a simple argument shows, this makes the sum over $x \in \mathbb{S}_{j} \backslash \Lambda_{j}$ finite uniformly in $\Lambda$ with a bounded aspect ratio. This concludes the proof.

\section{ACKNOWLEDGMENTS}

Part of this paper was written when M.B. was visiting Center for Theoretical Study in Prague. The research of R.K. was partly supported by the grants GAČR 201/03/0478 and MSM 110000001. The research of L.C. was supported by the NSF under the Grant DMS-9971016 and by the NSA under the Grant NSA-MDA 904-00-1-0050. R.K. would also like to thank the Max-Planck Institute for Mathematics in Leipzig for their hospitality as well as the A. von Humboldt Foundation whose Award made the stay in Leipzig possible.

\section{REFERENCES}

1. K. Alexander, J. T. Chayes, and L. Chayes, The Wulff construction and asymptotics of the finite cluster distribution for two-dimensional Bernoulli percolation, Comm. Math. Phys. 131:1-51 (1990).

2. K. Binder, Theory of evaporation/condensation transition of equilibrium droplets in finite volumes, Physica A 319:99-114 (2003).

3. K. Binder and M. H. Kalos, Critical clusters in a supersaturated vapor: Theory and Monte Carlo simulation, J. Statist. Phys. 22:363-396 (1980).

4. M. Biskup, L. Chayes, and R. Kotecký, On the formation/dissolution of equilibrium droplets, Europhys. Lett. 60:21-27 (2002).

5. M. Biskup, L. Chayes, and R. Kotecký, Critical region for droplet formation in the twodimensional Ising model, Comm. Math. Phys. 242:137-183 (2003).

6. T. Bodineau, The Wulff construction in three and more dimensions, Comm. Math. Phys. 207:197-229 (1999).

7. T. Bodineau, D. Ioffe, and Y. Velenik, Rigorous probabilistic analysis of equilibrium crystal shapes, J. Math. Phys. 41:1033-1098 (2000).

8. C. Borgs and R. Kotecký, Surface-induced finite-size effects for first-order phase transitions, J. Stat. Phys. 79:43-115 (1995).

9. J. Bricmont, J. L. Lebowitz, and C.-E. Pfister, On the local structure of the phase separation line in the two-dimensional Ising system, J. Stat. Phys. 26:313-332 (1981).

10. R. Cerf, Large deviations for three dimensional supercritical percolation, Astérisque 267: $\mathrm{vi}+177$ (2000). 
11. R. Cerf and A. Pisztora, On the Wulff crystal in the Ising model, Ann. Probab. 28:947-1017 (2000).

12. J. T. Chayes, L. Chayes, and R. H. Schonmann, Exponential decay of connectivities in the two-dimensional Ising model, J. Stat. Phys. 49:433-445 (1987).

13. P. Curie, Sur la formation des cristaux et sur les constantes capillaires de leurs différentes faces, Bull. Soc. Fr. Mineral. 8:145 (1885); Reprinted in Euvres de Pierre Curie (GauthierVillars, Paris, 1908), pp. 153-157.

14. R. L. Dobrushin, R. Kotecký, and S. B. Shlosman, Wulff Construction. A Global Shape from Local Interaction (Amer. Math. Soc., Providence, RI, 1992).

15. R. L. Dobrushin and S. B. Shlosman, in Probability Contributions to Statistical Mechanics (Amer. Math. Soc., Providence, RI, 1994), pp. 91-219.

16. H.-O. Georgii, Gibbs Measures and Phase Transitions, de Gruyter Studies in Mathematics, Vol. 9 (Walter de Gruyter, Berlin, 1988).

17. J. W. Gibbs, On the equilibrium of heterogeneous substances (1876), in Collected Works, Vol. 1 (Longmans, Green, 1928).

18. R. B. Griffiths, C. A. Hurst, and S. Sherman, Concavity of magnetization of an Ising ferromagnet in a positive external field, J. Math. Phys. 11:790-795 (1970).

19. D. Ioffe, Large deviations for the 2D Ising model: A lower bound without cluster expansions, J. Stat. Phys. 74:411-432 (1994).

20. D. Ioffe, Exact large deviation bounds up to $T_{\mathrm{c}}$ for the Ising model in two dimensions, Probab. Theory Related Fields 102:313-330 (1995).

21. D. Ioffe and R. H. Schonmann, Dobrushin-Kotecký-Shlosman theorem up to the critical temperature, Comm. Math. Phys. 199:117-167 (1998).

22. B. Krishnamachari, J. McLean, B. Cooper, and J. Sethna, Gibbs-Thomson formula for small island sizes: Corrections for high vapor densities, Phys. Rev. B 54:8899-8907 (1996).

23. R. Kotecký and D. Preiss, Cluster expansion for abstract polymer models, Comm. Math. Phys. 103:491-498 (1986).

24. L. D. Landau and E. M. Lifshitz, Course of theoretical physics, in Statistical Physics, Vol. 5 (Pergamon Press, Oxford/Edinburgh/New York, 1968).

25. T. Neuhaus and J. S. Hager, 2D crystal shapes, droplet condensation and supercritical slowing down in simulations of first order phase transitions, J. Stat. Phys. 113:47-83 (2003).

26. C.-E. Pfister, Large deviations and phase separation in the two-dimensional Ising model, Helv. Phys. Acta 64:953-1054 (1991).

27. C.-E. Pfister and Y. Velenik, Large deviations and continuum limit in the 2D Ising model, Probab. Theory Related Fields 109:435-506 (1997).

28. R. H. Schonmann and S. B. Shlosman, Wulff droplets and the metastable relaxation of kinetic Ising models, Comm. Math. Phys. 194:389-462 (1998).

29. D. Tabor, Gases, Liquids and Solids: And Other States of Matter, 3rd Edn. (Cambridge University Press, Cambridge, 1991).

30. G. Wulff, Zur Frage des Geschwindigkeit des Wachsturms und der Auflösung der Krystallflachen, Z. Krystallog. Mineral. 34:449-530 (1901). 